\title{
Relationship between Subtalar Joint Stiffness and Relaxed Calcaneal Stance Position in Cerebral Palsy Children with Valgus Deformities
}

\author{
Wei Chen, ${ }^{1}$ Jie Yao $\left(D,{ }^{1}\right.$ Yang Yang, ${ }^{1}$ Xiaoyu Liu,, ${ }^{1}$ Lizhen Wang, \\ Fang Pu (D), ${ }^{1}$ and Yubo Fan iD ${ }^{1,2}$ \\ ${ }^{1}$ Key Laboratory for Biomechanics and Mechanobiology of Ministry of Education, School of Biological Science and Medical Engineering, \\ Beijing Advanced Innovation Centre for Biomedical Engineering, Beihang University, Beijing, China \\ ${ }^{2}$ National Research Center for Rehabilitation Technical Aids, Beijing, China \\ Correspondence should be addressed to Fang Pu; pufangbme@buaa.edu.cn and Yubo Fan; yubofan@buaa.edu.cn
}

Received 13 October 2017; Revised 27 February 2018; Accepted 21 March 2018; Published 30 April 2018

Academic Editor: Cho-Pei Jiang

Copyright (C) 2018 Wei Chen et al. This is an open access article distributed under the Creative Commons Attribution License, which permits unrestricted use, distribution, and reproduction in any medium, provided the original work is properly cited.

\begin{abstract}
Relaxed calcaneal stance position (RCSP) is an important index in the correctional treatment of foot valgus deformities for cerebral palsy (CP) children. However, patients with similar RCSP showed diverse outcomes when accepting similar treatment, as the corrective resistance of subtalar joint (STJ) could be quite different. This study aimed to investigate the relationship between STJ stiffness and RCSP in different loading conditions. 38 valgus feet of 19 CP subjects were included in the study. A reposition force was applied beneath the STJ and pushed the foot from pronated position to neutral position. The STJ stiffness was calculated as the slope of the line fitting the force-displacement data. Correlations between the STJ stiffness, RCSP, and composite spasticity index (CSI) were analyzed. The spearman correlation coefficient indicated that STJ stiffness had no correlation with RCSPs, yet it had negative correlation with the change of RCSP under difference loading conditions $\left(\triangle \mathrm{RCSP}_{1 \mathrm{w}-0 \mathrm{w}}\right.$ and $\left.\Delta \mathrm{RCSP}_{0.5 \mathrm{w}-0 \mathrm{w}}\right)$. STJ stiffness was also correlated with the composite spasticity index (CSI), implying that this index had an advantage in reflecting the mechanism of valgus deformity and should be considered as a necessary measurement of foot valgus in CP children. The present method for quantification of STJ stiffness could improve the accuracy in the diagnosis and classification of foot deformity and may help increase the understanding of the biomechanical factors in foot deformity rehabilitation.
\end{abstract}

\section{Introduction}

Planovalgus is the most common foot deformity in cerebral palsy (CP) children [1-4]. It is characterized as hindfoot valgus during weight bearing, associated with a pronated midfoot and flattened longitudinal arch [5-7]. Long-term valgus deformity would lead to the navicular drop [8]. The head of the talus will become the main bearing areas and cause pain during walking $[1,9,10]$. The cause of valgus deformity is multifactorial, including soft tissue imbalance, muscle spasticity, and joint malalignment [10-12]. Therefore treatment method should be based on the inherent mechanism of the valgus deformity. Inappropriate treatment may lead to unsatisfactory outcome and complications $[6,13,14]$.
A precise assessment of the valgus severity usually plays an important role in the treatment options.

Relaxed calcaneal stance position (RCSP), radiographs, hindfoot valgus angle, and valgus index are commonly used methods to assess the foot valgus severity [5, 15-17]. These assessment indexes are based on the foot anatomical morphology under the static condition and have significant correlation with each other $[16,18]$. In particular, RCSP, defined as the angle between the heel bisection and a vertical line, is technically convenient and has been widely used in ankle deformity orthopedics $[19,20]$. However, there is still dissent regarding the efficiency of these morphological indexes, and the treatments based on these indexes also resulted in unsatisfactory outcomes [21]. 
Subtalar joint (STJ) stiffness is a biomechanical index that reflects the corrective resistance of the foot planovalgus. Clinicians have found that patients with similar morphological valgus could have very different corrective resistance [2224]. Treatment programs without considering the corrective resistance may fail to achieve adequate outcome. However, there is still a lack of quantitative definition of the corrective resistance. In manual reposition therapy, the corrective resistance is conventionally evaluated by experience. Interobserver reliability is low when inexperienced observers carry out the tests [25]. Our previous study has developed a method to quantify the apparent stiffness of subtalar joint (STJ), which could indicate the corrective resistance of the foot valgus deformity in CP children [26]. However, the relationship between the STJ stiffness and morphological valgus severity in CP children remains unclear. The insights gained through the relationship may lead a way to a better understanding of the mechanisms of the foot deformity and an optimization of the rehabilitation therapy.

This study aimed to investigate the relationship between the STJ stiffness and the morphological characteristics of valgus foot (RCSP) in CP children. Since there was no exact definition of STJ stiffness, the STJ stiffness was defined as the resistance in response to a reposition force, which was widely used for valgus deformity correction in CP children. To compare the STJ stiffness with RCSP from the perspective of foot deformity pathology, the correlations between the composite spasticity index (CSI) and the valgus parameters (STJ stiffness and RCSP) were also investigated.

\section{Materials and Methods}

2.1. Subjects. A total of 19 diplegic CP children (10 males and 9 females, age: $6.3 \pm 2.21$, height: $1.20 \pm 0.12 \mathrm{~m}$, and weight: $22.90 \pm 6.20 \mathrm{~kg}$ ) participated in this study. The inclusion criteria were as follows: (a) the spasticity was lower than level III according to the modified Ashworth scale [27]; (b) valgus deformity occurred at the hindfoot; (c) there was no surgery history of the foot or ankle; and (d) the heel could be manually flattened at the time of assessment. Informed written consent, in accordance with clinical protocols approved by Beihang University, was obtained from the parents of each subject.

2.2. STJ Stiffness Measurement. The STJ stiffness was measured with the device designed in our previous study (Figure 1) [26]. To standardize the position of the measurement, the subject was required to stand naturally on the platform; the ankle flexion angle was maintained at $90^{\circ}$. The foot was kept flat, and the lateral sides of foot were kept in contact with the fixed board. As shown in Figure 2, a reposition force was applied beneath the medial site of the talus with a horizontal angle of 40 degrees, which is a widely used reposition force for valgus deformity in CP children. The initial position of contact head was placed just in contact with the skin at the talus. Then the contact head pushed the STJ with a constant speed $(6 \mathrm{~mm} / \mathrm{s})$ to restore the foot from pronated position to neutral alignment. To make the reposition process more comfortable, the contact head of the

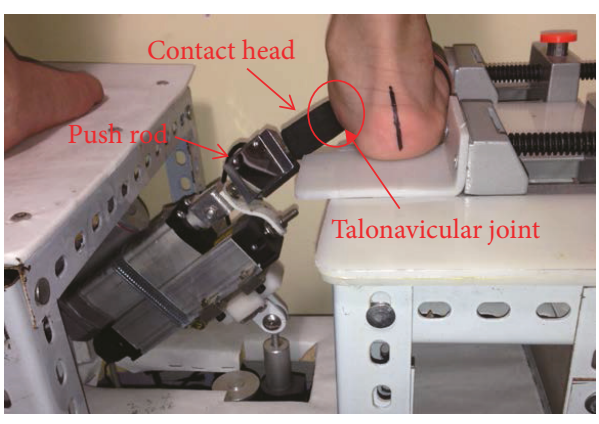

FIgURE 1: Overview of the device to measure the STJ stiffness.

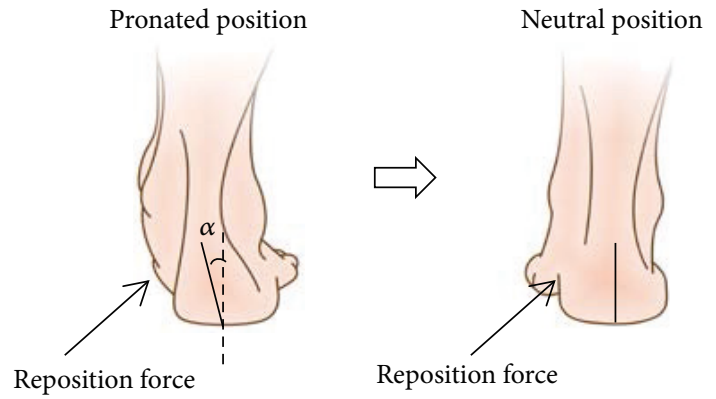

FIGURE 2: Schematic view of the measurement.

pushing rod was designed as a semicircular ethylene-vinyl acetate copolymer part. The force sensor (accuracy in $0.01 \mathrm{~kg}$, range in $200 \mathrm{~N}$, JLBS-v, System engineering Technology Co., Ltd., China) and the displacement sensor (sampling frequency in $12 \mathrm{~Hz}$, KTC-50 mm, Boshi Technology Co., Ltd., China) were fixed at the rod to record the force and displacement. Data were recorded in real time and transferred through a USB connection with a computer. Each foot was measured three times with 20-minute intervals. The forcedisplacement data was recorded.

2.3. RCSP Measurement. The subject was positioned in relaxed standing. The RCSP was defined as the angle between the calcaneal bisection line and the line perpendicular to the ground [19] ( $\alpha$ in Figure 2). Since RCSP will be influenced by the loading condition of the lower limbs, three conditions (weight-bearing, half-weight-bearing, and non-weightbearing) were considered in the experiment. In weightbearing condition, the subject stood with one foot, keeping the other foot backward off the ground, and the subject's hand could touch a handrail gently to balance himself; in half-weight-bearing condition, the subject stood in a relaxed bipedal stance with their feet apart as wide as an adult's fist; in non-weight-bearing condition, the subject sat with the feet fist-width apart, RCSP was measured on the unsupported feet. For each condition, the foot was measured three times with the protractor.

2.4. CSI Grading. Spasticity is a critical cause of valgus deformity, and CSI is a commonly used clinic index to assess the spasticity degree in CP children $[28,29]$. To compare the 
STJ stiffness and RCSP from the perspective of foot deformity pathology, CSI of each lower extremity was measured. The CSI subindexes (consist of Achilles tendon jerk, gastrocnemius tone, and ankle joint clonus) were graded, respectively.

2.5. Statistical Analysis. Spearman correlation matrices of STJ stiffness, RCSPs, and CSI were calculated by correlation analysis with the SPSS software (IMB, US). A correlation coefficient different from 0 and a significant level ( $p$ value) $<$ 0.05 indicate a considerable correlation. A two-way random average measure intraclass correlation coefficient $(\operatorname{ICC}(2, k))$ was used to assess the repeatability of the methodology [30]. A value greater than 0.75 indicates the desirable repeatability.

\section{Results}

3.1. CSI. The CSIs of 19 children with 38 feet were listed in Table 1. CSI is comprised of tendon jerk, muscle tone, and clonus. The mean score of tendon jerk was $2.4 \pm 0.59$. The mean score of muscle tone was $4.6 \pm 1.90$. The mean score of clonus was $1.2 \pm 0.36$. The mean CSI was $8.2 \pm 2.65$.

3.2. STJ Stiffness. The force-displacement data of the reposition process in one foot was shown in Figure 3(a). A least-square-fit line was calculated according to the forcedisplacement data. The STJ stiffness was defined as the slope of the line. The stiffness was 5.27, 5.24, and $5.35 \mathrm{~N} / \mathrm{mm}$, respectively, in three measurements of the foot. The accuracy of the fitting $\left(R^{2}\right)$ was $0.999,0.998$, and $0.998 \mathrm{~N}^{2}$, respectively. The STJ stiffness-CSI data was shown in Figure 3(b). The STJ stiffness was scattered within the range of $1.18 \mathrm{~N} / \mathrm{mm}$ to $7.73 \mathrm{~N} / \mathrm{mm}$. The mean stiffness of 38 feet was $2.94 \pm 1.27$ (mean $\pm \mathrm{SD})$.

3.3. RCSP. The RCSP decreased with the body weight loading (Figure 4(a)). The mean RCSP under weight-bearing condition (mean $\operatorname{RCSP}_{1 \mathrm{w}}$ ) was $15.91^{\circ} \pm 4.49^{\circ}$, under halfweight-bearing condition (mean $\mathrm{RCSP}_{0.5 \mathrm{w}}$ ) it was $11.06^{\circ} \pm$ $4.64^{\circ}$, and under non-weight-bearing condition (mean $\left.\operatorname{RCSP}_{0 \mathrm{w}}\right)$ it was $1.92^{\circ} \pm 4.61^{\circ}$. The RCSP-CSI data of 38 feet under three loading conditions were shown in Figures 4(b)-4(d). To further investigate the influence of loading alteration on the RCSP, the differences between $\operatorname{RCSP}_{1 \mathrm{w}}$ and $\operatorname{RCSP}_{0.5 \mathrm{w}}\left(\triangle \mathrm{RCSP}_{1 \mathrm{w}-0.5 \mathrm{w}}\right)$, between $\mathrm{RCSP}_{1 \mathrm{w}}$ and $\operatorname{RCSP}_{0 \mathrm{w}}\left(\triangle \mathrm{RCSP}_{1 \mathrm{w}-0 \mathrm{w}}\right)$, and between $\mathrm{RCSP}_{0.5 \mathrm{w}}$ and $\mathrm{RCSP}_{0 \mathrm{w}}$ $\left(\triangle \mathrm{RCSP}_{0.5 \mathrm{w}-0 \mathrm{w}}\right)$ were calculated (Figure 5).

The feet were further divided into two groups. $0^{\circ}<$ $\operatorname{RCSP}_{0.5 \mathrm{w}} \leq 10^{\circ}$ was classified as mild valgus group (group 1 ), and $10^{\circ}<\operatorname{RCSP}_{0.5 \mathrm{w}} \leq 20^{\circ}$ was classified as moderate valgus group (group 2) [31]. The mean STJ stiffness and $\triangle$ RCSPs were shown in Figure 6. With $\mathrm{RCSP}_{0.5 \mathrm{w}}$ increasing, mean STJ decreased and $\triangle \mathrm{RCSP}_{1 \mathrm{w}-0.5 \mathrm{w}}$ decreased, while $\triangle \mathrm{RCSP}_{1 \mathrm{w}-0 \mathrm{w}}$ and $\triangle \mathrm{RCSP}_{0.5 \mathrm{w}-0 \mathrm{w}}$ increased.

3.4. Correlation Analysis. The correlations between STJ stiffness, RCSPs, and $\triangle$ RCSPs were shown in Figure 7. STJ stiffness has no correlation with RCSPs and $\triangle \mathrm{RCSP}_{1 \mathrm{w}-0.5 \mathrm{w}}$ but has negative correlations with $\triangle \mathrm{RCSP}_{1 \mathrm{w}-0 \mathrm{w}}$ and $\Delta \mathrm{RCSP}_{0.5 \mathrm{w}-0 \mathrm{w}}$.
TABLE 1: CSIs of 19 children with 38 feet.

\begin{tabular}{|c|c|c|c|c|}
\hline Foot number & $\begin{array}{c}\text { Tendon jerk } \\
(0-4)\end{array}$ & $\begin{array}{l}\text { Muscle tone } \\
\quad(0-8)\end{array}$ & $\begin{array}{l}\text { Clonus } \\
(1-4)\end{array}$ & $\begin{array}{c}\text { CSI } \\
(1-16)\end{array}$ \\
\hline 1 & 2 & 4 & 1 & 7 \\
\hline 2 & 3 & 6 & 2 & 11 \\
\hline 3 & 2 & 4 & 1 & 7 \\
\hline 4 & 3 & 6 & 1 & 10 \\
\hline 5 & 2 & 4 & 1 & 7 \\
\hline 6 & 2 & 2 & 1 & 5 \\
\hline 7 & 2 & 4 & 1 & 7 \\
\hline 8 & 3 & 6 & 2 & 11 \\
\hline 9 & 3 & 6 & 1 & 10 \\
\hline 10 & 3 & 6 & 1 & 10 \\
\hline 11 & 2 & 4 & 1 & 7 \\
\hline 12 & 3 & 6 & 1 & 10 \\
\hline 13 & 2 & 2 & 1 & 5 \\
\hline 14 & 3 & 6 & 1 & 10 \\
\hline 15 & 2 & 2 & 1 & 5 \\
\hline 16 & 2 & 4 & 1 & 7 \\
\hline 17 & 2 & 4 & 1 & 7 \\
\hline 18 & 2 & 4 & 1 & 7 \\
\hline 19 & 2 & 2 & 1 & 5 \\
\hline 20 & 2 & 2 & 1 & 5 \\
\hline 21 & 3 & 6 & 1 & 10 \\
\hline 22 & 1 & 2 & 1 & 4 \\
\hline 23 & 2 & 4 & 1 & 7 \\
\hline 24 & 3 & 8 & 2 & 13 \\
\hline 25 & 3 & 6 & 1 & 10 \\
\hline 26 & 2 & 4 & 1 & 7 \\
\hline 27 & 3 & 8 & 2 & 13 \\
\hline 28 & 3 & 6 & 1 & 10 \\
\hline 29 & 2 & 2 & 1 & 5 \\
\hline 30 & 3 & 6 & 1 & 10 \\
\hline 31 & 1 & 2 & 1 & 4 \\
\hline 32 & 2 & 4 & 1 & 7 \\
\hline 33 & 3 & 8 & 2 & 13 \\
\hline 34 & 2 & 4 & 1 & 7 \\
\hline 35 & 3 & 8 & 2 & 13 \\
\hline 36 & 3 & 6 & 1 & 10 \\
\hline 37 & 2 & 2 & 1 & 5 \\
\hline 38 & 3 & 6 & 1 & 10 \\
\hline
\end{tabular}

A Spearman correlation matrix of all 8 variables was shown in Table 2. STJ stiffness was positively correlated with CSI $\left(r=0.343^{*} ; p=0.035\right)$. $\mathrm{RCSP}_{1 \mathrm{w}}, \mathrm{RCSP}_{0.5 \mathrm{w}}$, and $\mathrm{RCSP}_{0 \mathrm{w}}$ were correlated with $\triangle \mathrm{RCSP}_{1 \mathrm{w}-0 \mathrm{w}}$ and $\triangle \mathrm{RCSP}_{0.5 \mathrm{w}-0 \mathrm{w}}$. $\triangle \mathrm{RCSP}_{1 \mathrm{w}-0.5 \mathrm{w}}$ was not correlated with any other variables. Furthermore, the correlations between CSI subindexes (tendon jerk, muscle tone, and clonus) and the valgus indexes were shown in Table 3. The STJ stiffness was correlated with muscle tone $\left(r=0.335^{*} ; p=0.040\right)$ and clonus $\left(r=0.392^{*}\right.$; 


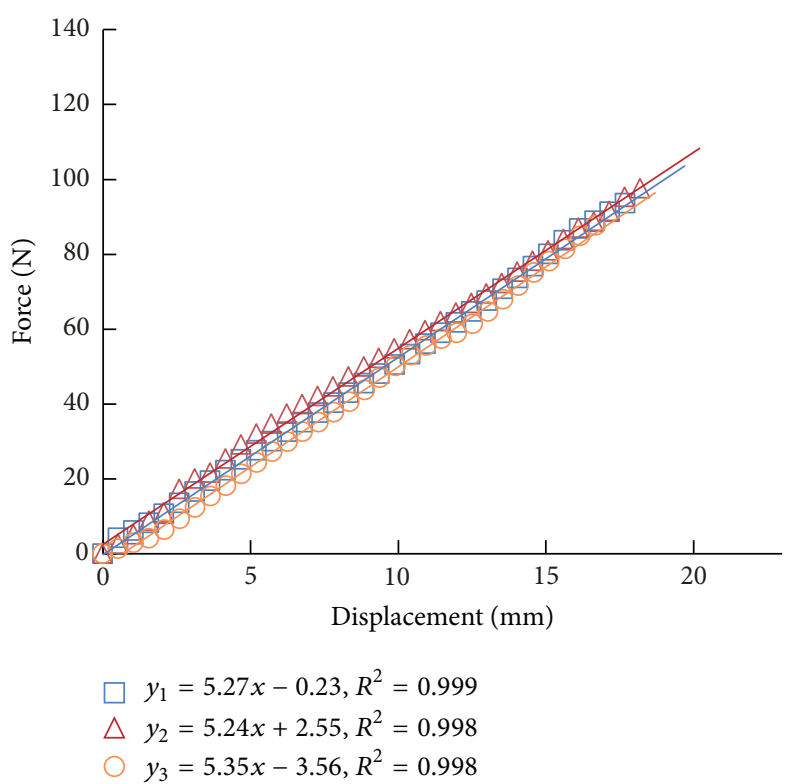

(a)

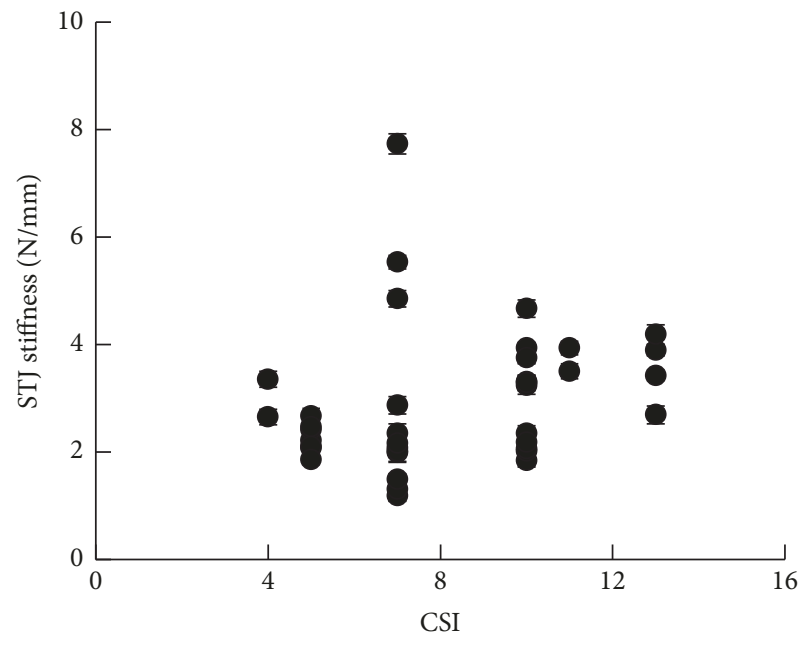

(b)

FiguRE 3: STJ stiffness of 38 feet. (a) Force-displacement data of reposition process in three measurements of one subject. A line was used to fit the force-displacement data with least squares fitting algorithm. The STJ stiffness was defined as the slope of the line. (b) STJ stiffness-CSI data of 38 feet.

$p=0.015)$. Tendon jerk was not correlated with any other variables. $\operatorname{ICC}(2, k)$ was 0.995 and the lower and upper bounds of the $95 \%$ confidence interval were 0.991 and 0.997 , which indicate desirable repeatability of the methodology.

\section{Discussion}

This study investigated the relationship between indexes of the foot valgus in spastic CP children, the STJ stiffness, and RCSPs. The character of STJ to resist deformation was contributed by talocalcaneal joint, talonavicular joint, and the related connective tissues. The STJ stiffness plays an important role in foot kinetics, especially during weightbearing activities. However, there was no exact definition of STJ stiffness previously. In the present study, the STJ stiffness was defined as the stiffness in response to an external force with a horizontal angle of 40 degrees, which was a widely used reposition force for valgus deformity in CP children. In the present study, CSI had a significant correlation with STJ stiffness $(r=0.343 ; p=0.035)$, whereas it had no correlation with RCSPs $\left(\mathrm{RCSP}_{1 \mathrm{w}}, \mathrm{RCSP}_{0.5 \mathrm{w}}\right.$, and $\left.\mathrm{RCSP}_{0 \mathrm{w}}\right)$. Since CSI is a reliable measure of lower extremity spasticity, which is one of the critical factors of foot valgus in CP children, the findings implied that STJ stiffness could be more directly related to the gait and foot function than RCSP.

Besides spasticity, STJ stiffness is also related to the abnormities in joint structure and tissue mechanical property. When the foot is at the weight-bearing position, a pathological STJ could lead to valgus deformity symptoms including the pronated position of the calcaneus, medial bulging of the navicular tuberosity, abduction of the forefoot, and a reduction in the height of the medial arch [32]. The degree of the STJ abnormities has a direct relationship with the severity of the foot deformity. Furthermore, the reposition ability of STJ under the external force was also considered as a reference for the development of treatment in clinics. However, this reposition ability was usually estimated with qualitative manual testing based on the therapists' experience $[22,25,33]$. The estimation accuracy is erratic and thus may lead to an unsatisfying treatment. The present study presented a quantitative method for the characterization of STJ stiffness, which could provide the basis for the development of treatment program.

RCSP was chosen as the morphological parameter to indicate the degree of foot deformity in this study, because the morphology-based indexes (e.g., RCSP, radiographs, valgus angle, and valgus index) have high correlations between each other $[16,18]$, and RCSP is more convenient to measure in clinics and has no risk of radiation. Compared to the STJ stiffness, RCSP is statically determined from the perspective of morphology. However, there is still dissent regarding the efficiency of morphological indexes; the treatments based on morphological indexes also resulted in unsatisfactory outcomes [21]. The present study divided the feet into mild and moderate valgus groups according to the degree of RCSP; the results showed that the mean STJ stiffness in mild valgus group was even greater than that in moderate valgus group. The present study further demonstrated that the RCSPs $\left(\mathrm{RCSP}_{1 \mathrm{w}}, \mathrm{RCSP}_{0.5 \mathrm{w}}\right.$, and $\mathrm{RCSP}_{0 \mathrm{w}}$ ) had no significant relationship with STJ stiffness. Although some patients' RCSPs are similar, their joint mechanical properties relevant to the reposition ability may be diverse (e.g., muscular spasticity and ligamentous flexibility) [34]. The present findings potentially implied that RCSPs might not be efficiently correlated with 


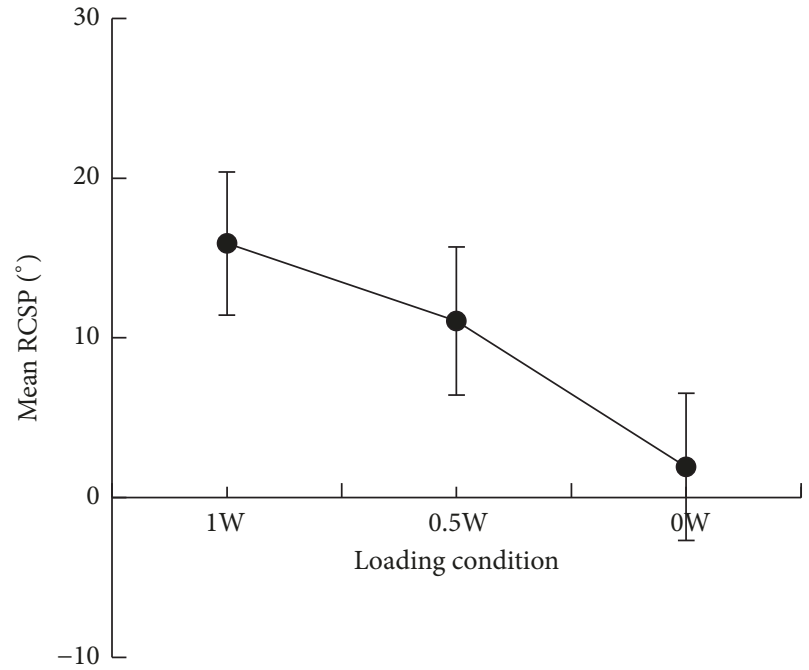

(a)

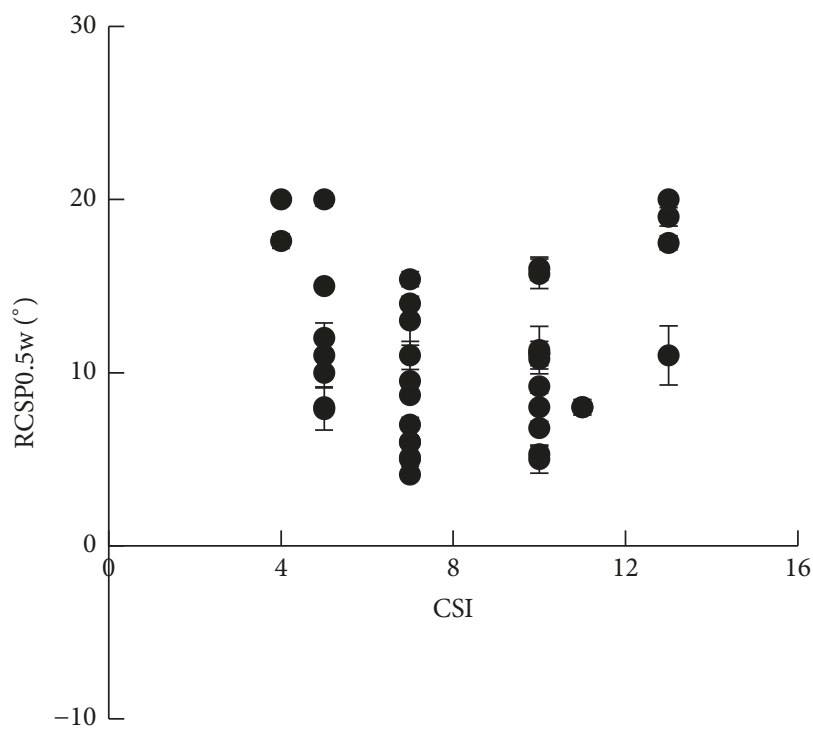

(c)

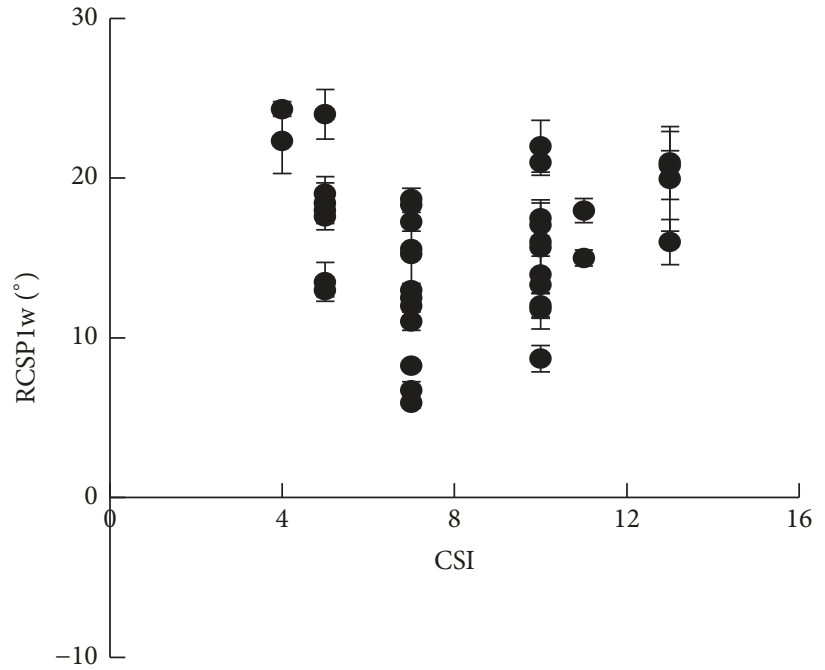

(b)

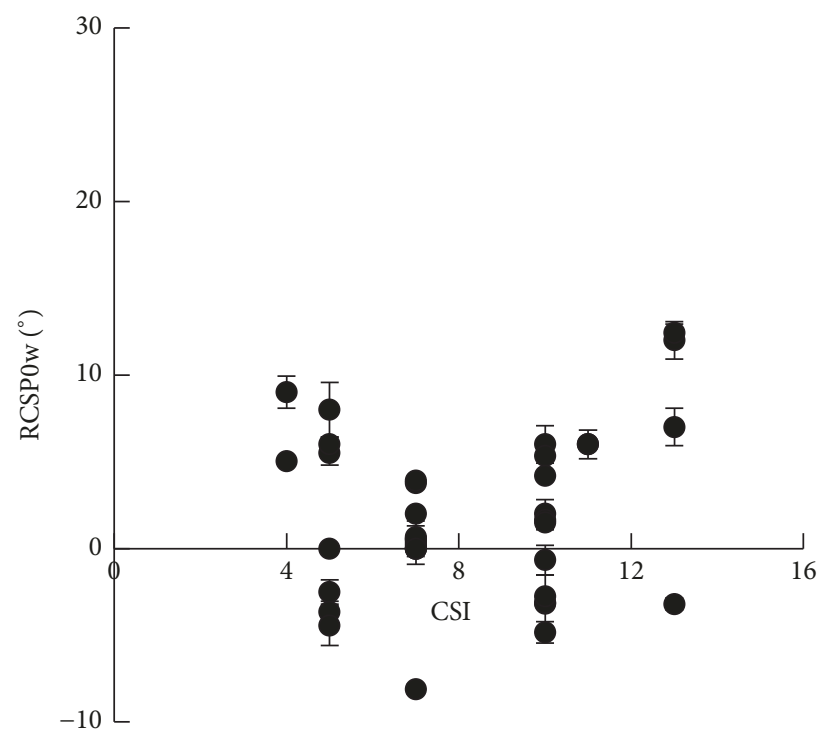

(d)

FIgURE 4: RCSP of 38 feet. (a) The mean RCSP decreased with the body weight loading. (b) The RCSP ${ }_{1 w}$-CSI data. (c) The RCSP ${ }_{0.5 w}$-CSI data. (d) The RCSP $_{0 \mathrm{w}}$-CSI data.

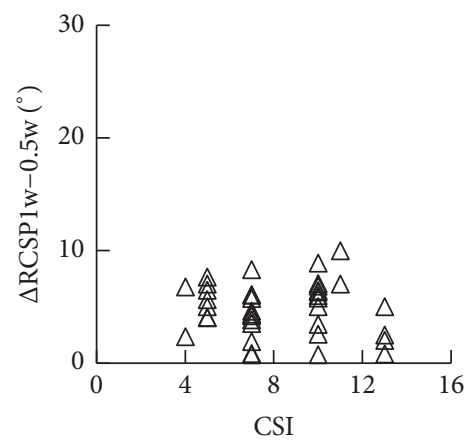

(a)

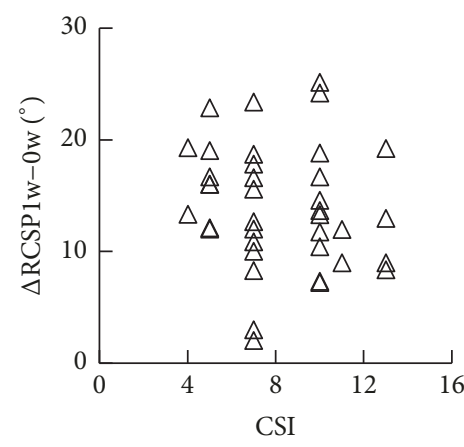

(b)

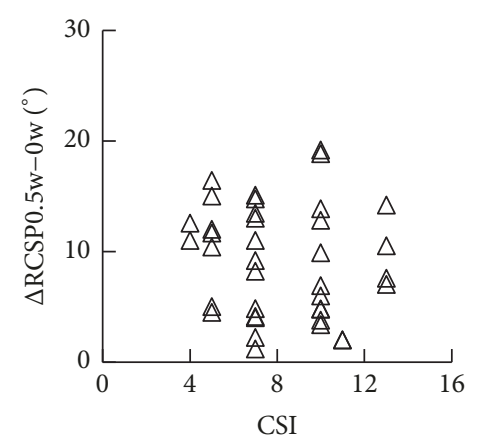

(c)

FIGURE 5: Influence of loading condition on RCSP. (a) $\Delta \operatorname{RCSP}_{1 \mathrm{w}-0.5 \mathrm{w}}=\operatorname{RCSP}_{1 \mathrm{w}}-\mathrm{RCSP}_{0.5 \mathrm{w}}$. (b) $\Delta \mathrm{RCSP}_{1 \mathrm{w}-0 \mathrm{w}}=\operatorname{RCSP}_{1 \mathrm{w}}-\mathrm{RCSP}_{0 \mathrm{w}}$. (c) $\Delta \mathrm{RCSP}_{0.5 \mathrm{w}-0 \mathrm{w}}=\mathrm{RCSP}_{0.5 \mathrm{w}}-\mathrm{RCSP}_{0 \mathrm{w}}$. 


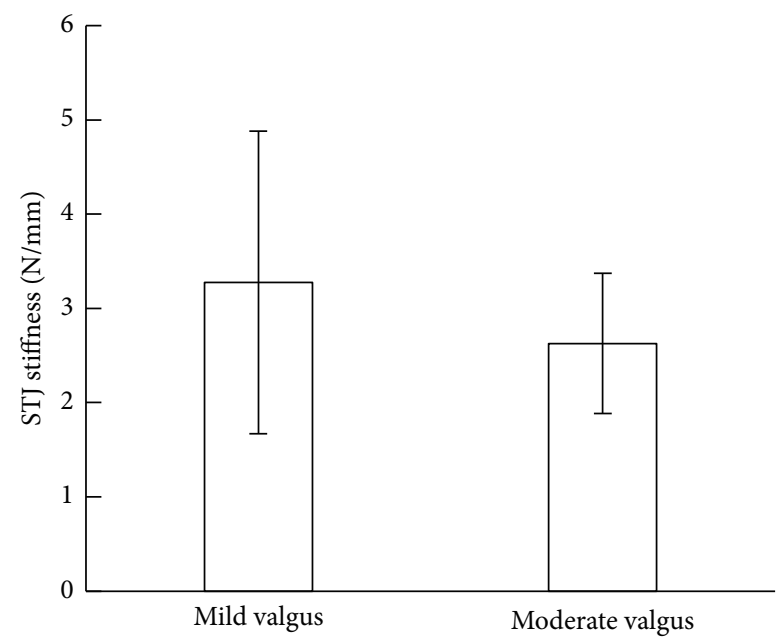

(a)

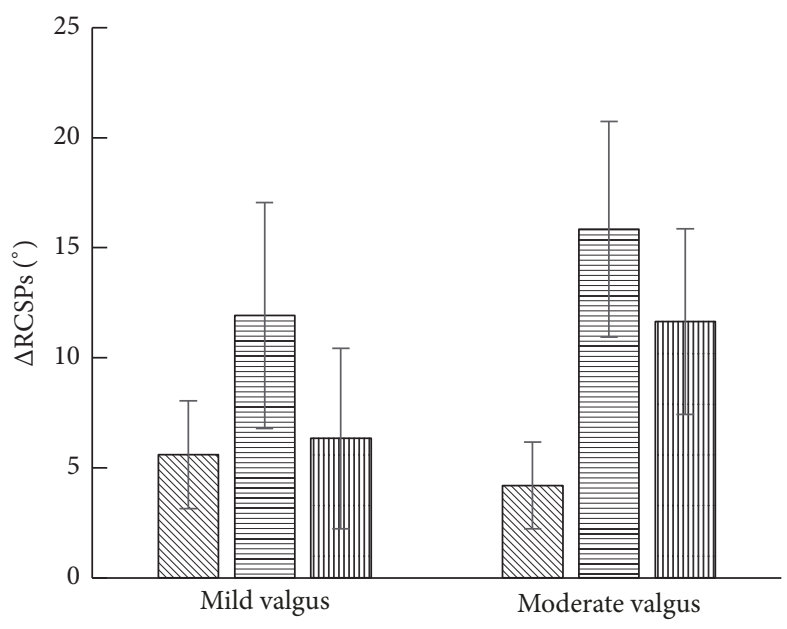

$\mathbb{Q} \Delta \mathrm{RCSP} 1 \mathrm{w}-0.5 \mathrm{w}$
目 $\Delta$ RCSP $1 \mathrm{w}-0 \mathrm{w}$
血 $\Delta$ RCSP $0.5 \mathrm{w}-0 \mathrm{w}$

Figure 6: STJ stiffness and $\triangle$ RCSPs in mild and moderate valgus groups. (a) Mean STJ stiffness. (b) Mean $\Delta \mathrm{RCSP}_{1 \mathrm{w}-0.5 \mathrm{w}}, \Delta \mathrm{RCSP} \mathrm{lw}_{\mathrm{w}-0 \mathrm{w}}$, and $\triangle \mathrm{RCSP}_{0.5 \mathrm{w}-0 \mathrm{w}}$.

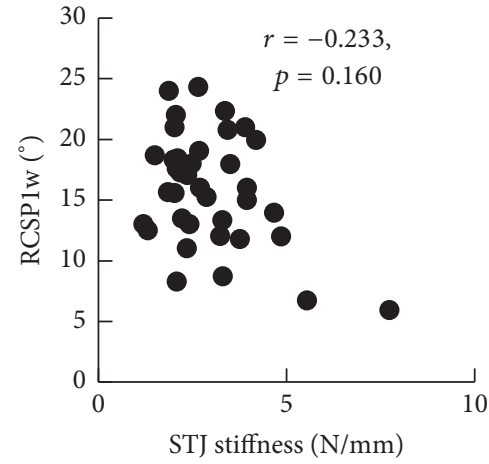

(a)

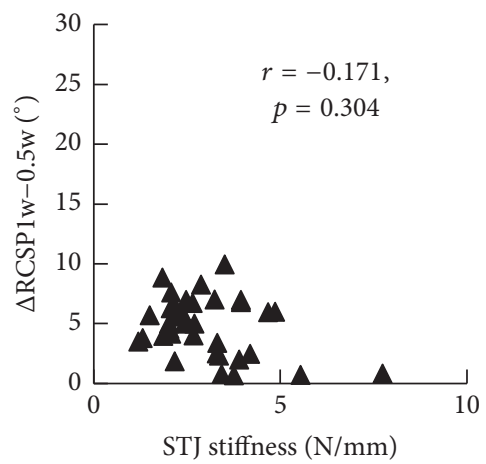

(d)

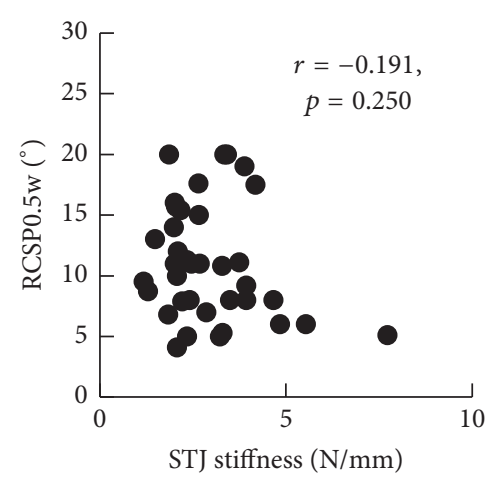

(b)

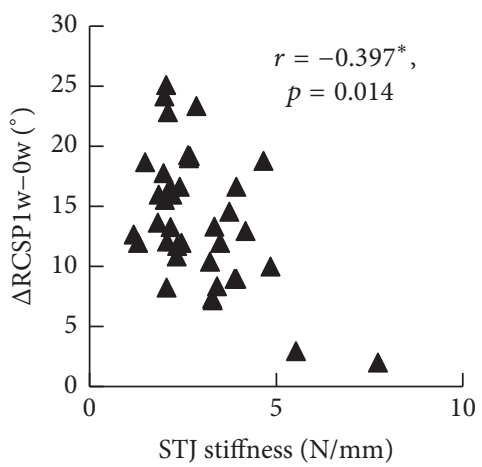

(e)

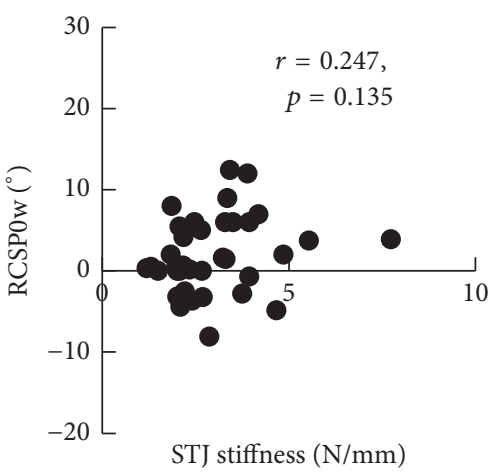

(c)

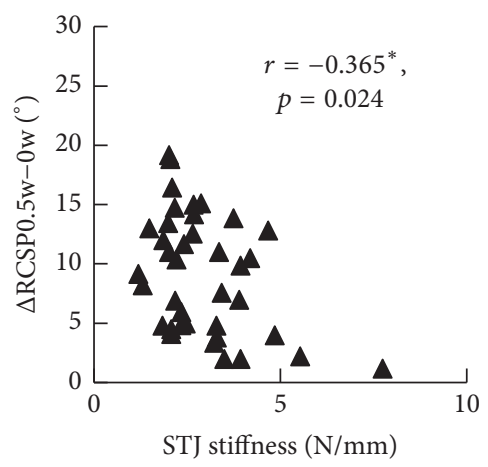

(f)

FiguRE 7: Correlations between STJ stiffness, RCSPs, and $\Delta$ RCSPs. (a) Correlation between STJ stiffness and RCSP lw $(r=-0.233 ; p=0.160$ ). (b) Correlation between STJ stiffness and $\operatorname{RCSP}_{0.5 \mathrm{w}}(r=-0.191 ; p=0.250)$. (c) Correlation between STJ stiffness and RCSP $\mathrm{Rw}(r=0.247$; $p=0.135)$. (d) Correlation between STJ stiffness and $\Delta \operatorname{RCSP}_{1 \mathrm{w}-0.5 \mathrm{w}}(r=-0.171 ; p=0.304)$. (e) Correlation between STJ stiffness and $\Delta \mathrm{RCSP}_{1 \mathrm{w}-0 \mathrm{w}}\left(r=-0.397^{*} ; p=0.014\right)$. (f) Correlation between STJ stiffness and $\Delta \mathrm{RCSP}_{0.5 \mathrm{w}-0 \mathrm{w}}\left(r=-0.365^{*} ; p=0.024\right)$. 


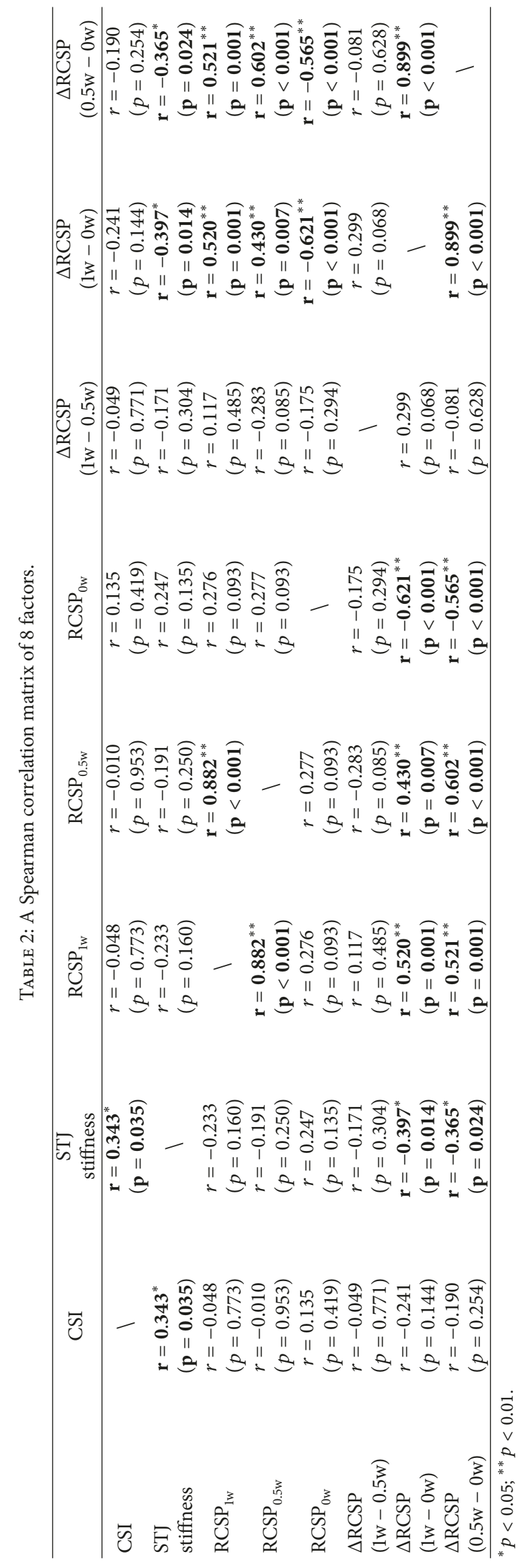


TABLE 3: Correlations between CSI subindexes and the valgus indexes.

\begin{tabular}{llllllll}
\hline & $\begin{array}{c}\text { STJ } \\
\text { Stiffness }\end{array}$ & $\mathrm{RCSP}_{1 \mathrm{w}}$ & $\mathrm{RCSP}_{0.5 \mathrm{w}}$ & $\mathrm{RCSP}_{0 \mathrm{w}}$ & $\begin{array}{c}\Delta \mathrm{RCSP} \\
(1 \mathrm{w}-0.5 \mathrm{w})\end{array}$ & $\begin{array}{c}\Delta \mathrm{RCSP} \\
(1 \mathrm{w}-0 \mathrm{w})\end{array}$ & $\begin{array}{c}\Delta \mathrm{RCSP} \\
(0.5 \mathrm{w}-0 \mathrm{w})\end{array}$ \\
\hline \multirow{2}{*}{ Tendon jerk } & $r=0.284$ & $r=-0.009$ & $r=-0.005$ & $r=0.060$ & $r=0.085$ & $r=-0.137$ & $r=-0.139$ \\
& $(p=0.084)$ & $(p=0.960)$ & $(p=0.975)$ & $(p=0.720)$ & $(p=0.611)$ & $(p=0.413)$ & $(p=0.406)$ \\
Muscle tone & $r=0.335^{*}$ & $r=-0.034$ & $r=0.026$ & $r=0.121$ & $r=-0.090$ & $r=-0.215$ & $r=-0.146$ \\
& $(p=0.040)$ & $(p=0.839)$ & $(p=0.878)$ & $(p=0.469)$ & $(p=0.590)$ & $(p=0.194)$ & $(p=0.381)$ \\
Clonus & $r=0.392^{*}$ & $r=0.257$ & $r=0.224$ & $r=0.395^{*}$ & $r=-0.069$ & $r=-0.211$ & $r=-0.165$ \\
& $(p=0.015)$ & $(p=0.120)$ & $(p=0.176)$ & $(p=0.014)$ & $(p=0.680)$ & $(p=0.204)$ & $(p=0.324)$ \\
\hline
\end{tabular}

${ }^{*} p<0.05$.

the mechanism of foot deformity in CP children and therefore not suitable as the primary principle for the development of treatment.

Although RCSP is a morphological index under a loading condition, $\triangle \mathrm{RCSP}$ could indicate the morphological change under different loadings, which means that $\triangle$ RCSP could partially reflect the ankle stiffness in the coronal plane of the heel. The results indicated that STJ stiffness had significant negative correlations with $\triangle \mathrm{RCSP} 1 \mathrm{w}-0 \mathrm{w}(-0.397, p=0.014)$ and $\triangle \mathrm{RCSP} 0.5 \mathrm{w}-0 \mathrm{w}(-0.365, p=0.024)$. The correlation factors were small, because $\triangle \mathrm{RCSP}$ reflected the calcaneal deformation in response to the vertical force on pelma, yet STJ stiffness reflected the calcaneal deformation in response to the reposition force.

The force-displacement curve was collected during the reposition process. The curve slope was small in the initial stage of loading; the small resistance in this stage was mainly contributed by the superficial skin and fat. With increasing displacement, the resistance rose linearly, which was contributed by the STJ structure and mechanical property. In the present study, STJ stiffness was defined as the slope of the line fitting the force-displacement curve, because the initial stage was short and had little influence on the calculated stiffness.

Although significant correlation was observed between STJ stiffness and CSI, only muscle tone and clonus had correlation with STJ stiffness. Previous study has reported that the muscle tone and muscle stiffness in CP children were higher than those in normal children [35]. Clonus is involuntary and rhythmic muscle contractions caused by a permanent lesion in upper motor neurons [36]. Therefore, both the muscle tone and clonus could increase the STJ constraint and contribute to the joint stiffness.

The present study has some limitations. First, in the process of measuring the STJ stiffness, the reposition force may influence the balance of the subject and influence the force-displacement data. However, the valgus deformities in this study were at mild and moderate level; the reposition forces were relatively small and had slight influence on the results. An improvement of the method is still necessary to decrease the potential deviation in our future study. Secondary, since the subjects were young children, the degree of their cooperation would also affect the measurements.

\section{Conclusion}

The present study quantified two indexes of the foot valgus in 38 feet of spastic CP children, the STJ stiffness and RCSPs (in three loading conditions). STJ stiffness had no correlation with RCSPs, yet it had negative correlation with the change of RCSP under different loading conditions $\left(\triangle \mathrm{RCSP}_{1 \mathrm{w}-0 \mathrm{w}}\right.$ and $\left.\triangle \mathrm{RCSP}_{0.5 \mathrm{w}-0 \mathrm{w}}\right)$. STJ stiffness was also correlated with the composite spasticity index (CSI), implying that this index had an advantage in reflecting the mechanism of valgus deformity and should be considered as a necessary measurement of foot valgus in $\mathrm{CP}$ children. The present method for quantification of STJ stiffness could improve the accuracy in the diagnosis and classification of foot deformity and may help increase the understanding of the biomechanical factors in foot deformity rehabilitation.

\section{Disclosure}

Wei Chen and Jie Yao are co-first authors.

\section{Conflicts of Interest}

There are no conflicts of interest regarding the publication of this study.

\section{Acknowledgments}

The authors would like to convey their appreciation to Dr. Yang Chen of Peking Union Medical College Hospital for the very kind suggestion. This study was supported by the National Natural Science Foundation of China (nos. 11502014,11421202 , and 11672027), the Open Foundation of State Key Laboratory of Space Medicine Fundamentals and Application (SMFA16K01), Young Elite Scientist Sponsorship Program by CAST (YESS 2015QNRC001), National Key Research and Development Program (2016YFC1101904 and 2016YFB1101101), the National Key Lab of Virtual Reality Technology, and Defense Industrial Technology Development Program (JCKY2016601B009). The authors thank all the subjects who participated in the research and acknowledge Rokab Pedorthic Center for the help in collecting patients.

\section{References}

[1] M. Kadhim, L. Holmes Jr., C. Church, J. Henley, and F. Miller, "Pes planovalgus deformity surgical correction in ambulatory children with cerebral palsy," Journal of Children's Orthopaedics, vol. 6, no. 3, pp. 217-227, 2012. 
[2] C.-A. Luo, H.-K. Kao, W.-C. Lee, W.-E. Yang, and C.-H. Chang, "Limits of calcaneal lengthening for treating planovalgus foot deformity in children with cerebral palsy," Foot \& Ankle International, vol. 38, no. 8, pp. 863-869, 2017.

[3] M. Kadhim and F. Miller, "Pes planovalgus deformity in children with cerebral palsy: review article," Journal of Pediatric Orthopaedics B, vol. 23, no. 5, pp. 400-405, 2014.

[4] M. S. Karamitopoulos and L. Nirenstein, "Neuromuscular foot: spastic cerebral palsy," Foot and Ankle Clinics, vol. 20, no. 4, pp. 657-668, 2015.

[5] M. Kadhim, L. Holmes, and F. Miller, "Correlation of radiographic and pedobarograph measurements in planovalgus foot deformity," Gait \& Posture, vol. 36, no. 2, pp. 177-181, 2012.

[6] L. B. Gehrig, "CORR insights ${ }^{\circledR}$ : incidence and risk factors of allograft bone failure after calcaneal lengthening," Clinical Orthopaedics and Related Research, vol. 473, no. 5, pp. 1775-1776, 2015.

[7] K. M. Kruger, K. A. Konop, J. J. Krzak et al., "Segmental kinematic analysis of planovalgus feet during walking in children with cerebral palsy," Gait \& Posture, vol. 54, pp. 277-283, 2017.

[8] J. A. Heydemann, O. Abousamra, J. M. Franzone, B. E. Kaufman, and J. P. Sees, "What's new in the management of foot deformities in children with cerebral palsy," Journal of Pediatric Orthopaedics, vol. 38, no. 1, pp. e20-e24, 2018.

[9] K. B. Park, H. W. Park, K. S. Lee, S. Y. Joo, and H. W. Kim, "Changes in dynamic foot pressure after surgical treatment of valgus deformity of the hindfoot in cerebral palsy," The Journal of Bone \& Joint Surgery-American Volume, vol. 90, no. 8, pp. 1712-1721, 2008.

[10] P. Kedem and D. M. Scher, "Foot deformities in children with cerebral palsy," Current Opinion in Pediatrics, vol. 27, no. 1, pp. 67-74, 2015.

[11] C. Church, N. Lennon, R. Alton et al., "Longitudinal change in foot posture in children with cerebral palsy," Journal of Children's Orthopaedics, vol. 11, no. 3, pp. 229-236, 2017.

[12] C. Boulay, M. Jacquemier, E. Castanier et al., "Planovalgus foot deformity in cerebral palsy corrected by botulinum toxin injection in the peroneus longus: clinical and radiological evaluations in young children," Annals of Physical and Rehabilitation Medicine, vol. 58, no. 6, pp. 316-321, 2015.

[13] J. P. Sees and F. Miller, "Overview of foot deformity management in children with cerebral palsy," Journal of Children's Orthopaedics, vol. 7, no. 5, pp. 373-377, 2013.

[14] B. C. Cho, I. H. Lee, C. Y. Chung et al., "Undercorrection of planovalgus deformity after calcaneal lengthening in patients with cerebral palsy," Journal of Pediatric Orthopaedics B, vol. 27, no. 3, pp. 206-213, 2018.

[15] B. Leidinger, T. J. Heyse, S. Fuchs-Winkelmann, J. R. J. Paletta, and R. Roedl, "Grice-Green procedure for severe hindfoot valgus in ambulatory patients with cerebral palsy," Journal of Foot and Ankle Surgery, vol. 50, no. 2, pp. 190-196, 2011.

[16] B. M. Lamm, R. W. Mendicino, A. R. Catanzariti, and H. J. Hillstrom, "Static rearfoot alignment: A comparison of clinical and radiographic measures," Journal of the American Podiatric Medical Association, vol. 95, no. 1, pp. 26-33, 2005.

[17] E. Billis, E. Katsakiori, C. Kapodistrias, and E. Kapreli, "Assessment of foot posture: Correlation between different clinical techniques," The Foot, vol. 17, no. 2, pp. 65-72, 2007.

[18] J. S. Lee, K. B. Kim, J. O. Jeong, N. Y. Kwon, and S. M. Jeong, "Correlation of foot posture index with plantar pressure and radiographic measurements in pediatric flatfoot," Annals of Rehabilitation Medicine, vol. 39, no. 1, pp. 10-17, 2015.
[19] S. Y. Ahn, S. K. Bok, B. O. Kim, and I. S. Park, "The effects of talus control foot orthoses in children with flexible flatfoot," Journal of the American Podiatric Medical Association, vol. 107, no. 1, pp. 46-53, 2017.

[20] H.-J. Lee, K.-B. Lim, J. Yoo, S.-W. Yoon, and T.-H. Jeong, "Effect of foot orthoses on children with lower extremity growing pains," Annals of Rehabilitation Medicine, vol. 39, no. 2, pp. 285293, 2015.

[21] A. Jane MacKenzie, K. Rome, and A. M. Evans, "The efficacy of nonsurgical interventions for pediatric flexible flat foot: A critical review," Journal of Pediatric Orthopaedics, vol. 32, no. 8, pp. 830-834, 2012.

[22] C. Payne, S. Munteanu, and K. Miller, "Position of the subtalar joint axis and resistance of the rearfoot to supination.," Journal of the American Podiatric Medical Association, vol. 93, no. 2, pp. 131-135, 2003.

[23] K. A. Kirby, "Subtalar joint axis location and rotational equilibrium theory of foot function," Journal of the American Podiatric Medical Association, vol. 91, no. 9, pp. 465-487, 2001.

[24] I. B. Griffiths and I. M. McEwan, "Reliability of a new supination resistance measurement device and validation of the manual supination resistance test," Journal of the American Podiatric Medical Association, vol. 102, no. 4, pp. 278-289, 2012.

[25] H. Noakes and C. Payne, "The reliability of the manual supination resistance test.", Journal of the American Podiatric Medical Association, vol. 93, no. 3, pp. 185-189, 2003.

[26] W. Chen, F. Pu, Y. Yang, J. Yao, L. Wang, and Y. Fan, "Quantitative measurement of subtalar joint passive stiffness in children with cerebral palsy," in Proceedings of the World Congress on Medical Physics and Biomedical Engineering, pp. 1132-1136, Toronto, Canada, 2015.

[27] B. C. Craven and A. R. Morris, "Modified ashworth scale reliability for measurement of lower extremity spasticity among patients with SCI," Spinal Cord, vol. 48, no. 3, pp. 207-213, 2010.

[28] A. I. Ibrahim and Z. M. Hawamdeh, "Evaluation of physical growth in cerebral palsied children and its possible relationship with gross motor development," International Journal of Rehabilitation Research, vol. 30, no. 1, pp. 47-54, 2007.

[29] M. F. Levin and C. W. Y. Hui-Chan, "Relief of hemiparetic spasticity by TENS is associated with improvement in reflex and voluntary motor functions," Electroencephalography and Clinical Neurophysiology, vol. 85, no. 2, pp. 131-142, 1992.

[30] S. Rao, J. Song, A. Kraszewski et al., "The effect of foot structure on 1st metatarsophalangeal joint flexibility and hallucal loading," Gait \& Posture, vol. 34, no. 1, pp. 131-137, 2011.

[31] K. E. Sell, T. M. Verity, T. W. Worrell, B. J. Pease, and J. Wigglesworth, "Two measurement techniques for assessing subtalar joint position: A reliability study," Journal of Orthopaedic \& Sports Physical Therapy, vol. 19, no. 3, pp. 162-167, 1994.

[32] R. Donatelli, "Abnormal biomechanics of the foot and ankle," Journal of Orthopaedic \& Sports Physical Therapy, vol. 9, no. 1, pp. 11-16, 1987.

[33] A. D. Cass and C. A. Camasta, "A review of tarsal coalition and pes planovalgus: clinical examination, diagnostic imaging, and surgical planning," The Journal of Foot and Ankle Surgery, vol. 49, no. 3, pp. 274-293, 2010.

[34] A. Faria, R. Gabriel, J. Abrantes, R. Brás, and H. Moreira, "The relationship of body mass index, age and triceps-surae musculotendinous stiffness with the foot arch structure of postmenopausal women," Clinical Biomechanics, vol. 25, no. 6, pp. 588-593, 2010. 
[35] G.-M. Um, J.-S. Wang, and S.-E. Park, "An analysis on muscle tone of lower limb muscles on flexible flat foot," Journal of Physical Therapy Science, vol. 27, no. 10, pp. 3089-3092, 2015.

[36] I. Boyraz, H. Uysal, B. Koc, and H. Sarman, "Clonus: definition, mechanism, treatment," Medicinski Glasnik: Official Publication of The Medical Association of Zenica-Doboj Canton, Bosnia And Herzegovina, vol. 12, no. 1, 19 pages, 2015. 


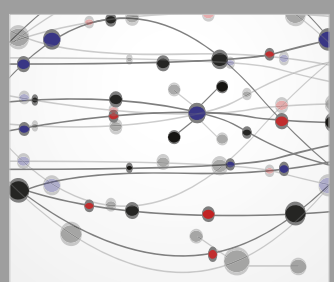

The Scientific World Journal
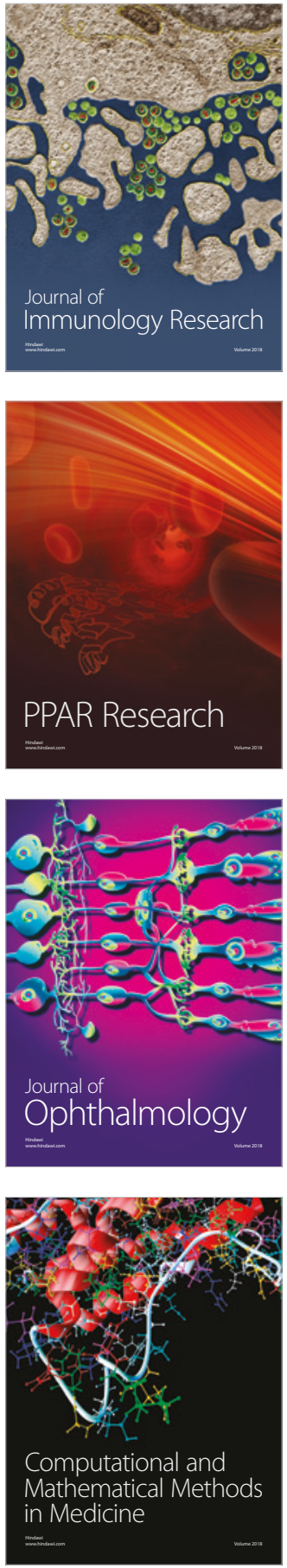

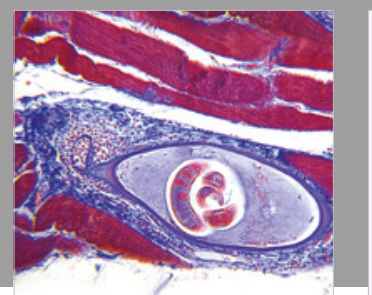

Gastroenterology Research and Practice

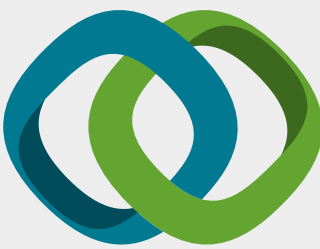

\section{Hindawi}

Submit your manuscripts at

www.hindawi.com
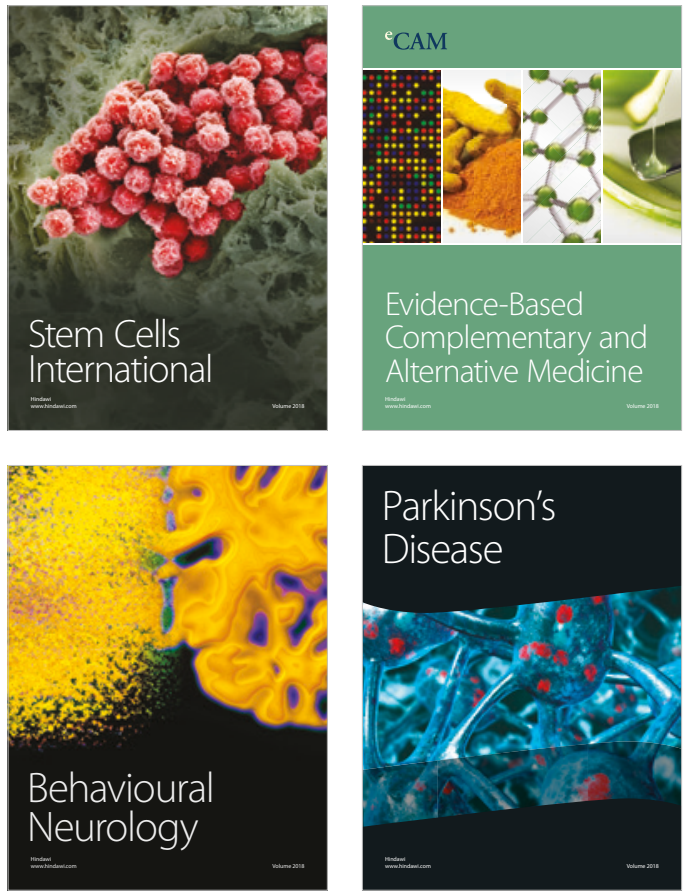

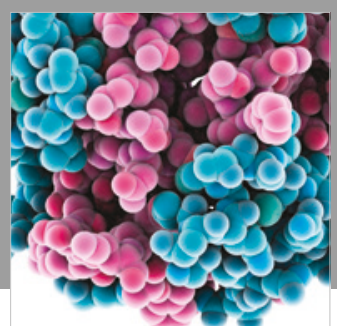

ournal of

Diabetes Research

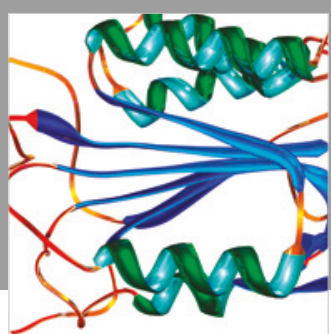

Disease Markers
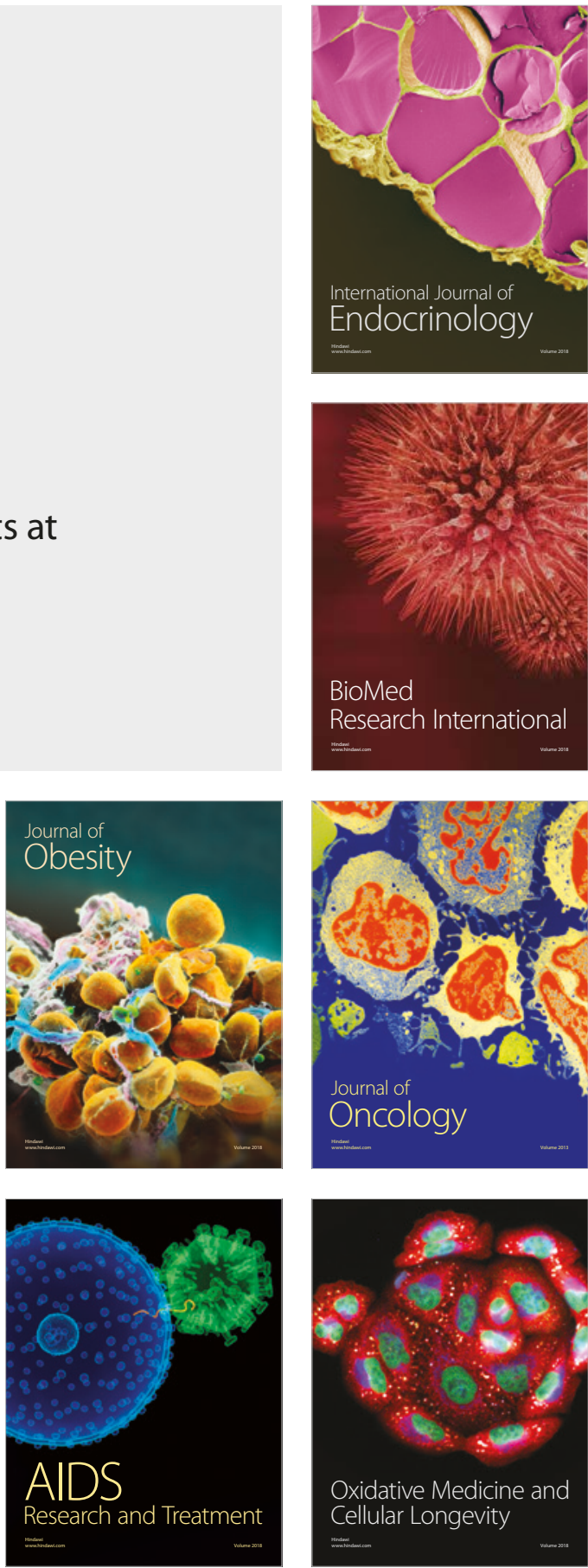